\title{
MÉTODOS DE OBTENÇÃO, REATIVIDADE E IMPORTÂNCIA BIOLÓGICA DE 4-TIAZOLIDINONAS
}

André P. Liesen, Thiago M. de Aquino e Alexandre J. S. Góes*

Departamento de Antibióticos, Universidade Federal de Pernambuco, Cidade Universitária, 50670-901 Recife - PE, Brasil

José G. de Lima, Antônio R. de Faria e Antonio J. Alves

Departamento de Ciências Farmacêuticas, Universidade Federal de Pernambuco, Cidade Universitária, 50470-521 Recife - PE, Brasil

Recebido em 23/10/06; aceito em 21/6/07; publicado na web em 26/2/08

PREPARATION METHODS, REACTIVITY AND BIOLOGICAL IMPORTANCE OF 4-THIAZOLIDINONES. Molecules containing the 4-thiazolidinone ring are known to possess a wide range of biological properties including antimicrobial and antiinflammatory activities among others. These compounds can be synthesized by cyclization reactions involving $\alpha$-haloacetic acid or $\alpha$-mercaptoacetic acid and employed in several chemoselective reactions. Comprehensive reviews have been written on 4thiazolidinones in 1961 by Brown and in 1980 by Singh et al. In the recent literature, some new synthesis methods for 4thiazolidinone derivatives and several reactions have been reported. These advances warrant to review the chemical and biological properties of compounds with this important heterocycle employed in synthetic organic chemistry and medicinal chemistry.

Keywords: 4-thiazolidinone; preparation methods; reactivity.

\section{INTRODUÇÃO}

Nos últimos 20 anos houve um aumento alarmante da resistência de microrganismos - especialmente bactérias Gram-positivas à ação de medicamentos ${ }^{1-5}$. Este fato observado pode ser atribuído à mutagenicidade destes agentes patógenos, provocada pela administração de múltiplos fármacos em pacientes hospitalizados, e em pacientes imunocomprometidos com câncer, AIDS ou transplanta$\operatorname{dos}^{2,6}$.

A enzima enolpiruvil uridina difosfato $N$-acetilglucosamina redutase (Mur B), uma flavoproteína existente apenas em seres procariontes, tem papel fundamental na biossíntese do polímero peptidoglicano ${ }^{7}$. Este biopolímero extracelular é essencial para a manutenção da integridade osmótica da parede celular bacteriana, tanto em microrganismos Gram-positivos como em Gram-negati$\operatorname{vos}^{8}$. O peptidoglicano é constituído pela alternância de dois aminoaçúcares - $N$-acetil-glucosamina $(\mathrm{G})$ e ácido $N$-acetil- murâmico (M) - acoplados a um tetra ou pentapeptídio contendo o fragmento lisina ou diaminopimelato9. A principal etapa da biossíntese deste polímero é a formação do precursor ácido uridina difosfato $\mathrm{N}$ acetilmurâmico (UNAM), por ser originado deste intermediário o grupo lactil-éter, responsável pelo acoplamento entre o dissacarídeo e a porção peptídica e, conseqüentemente, pela formação da unidade monomérica do peptidoglicano ${ }^{8}$. A ação da enzima Mur B consiste na redução da porção enol-éter, do intermediário enolpiruvil uridina difosfato $N$-acetilglucosamina (EP-UNAG), à porção lactil-

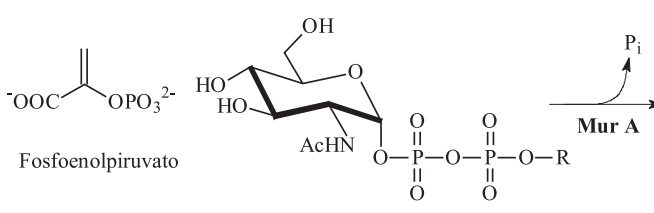

$N$-acetilglucosamina

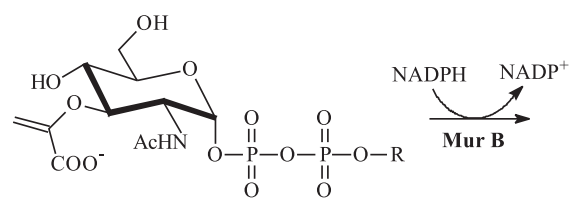

EP-UNAG

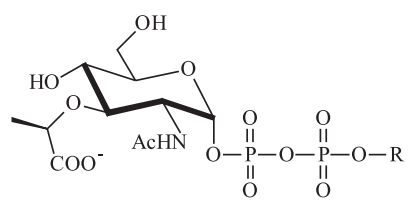

UNAM

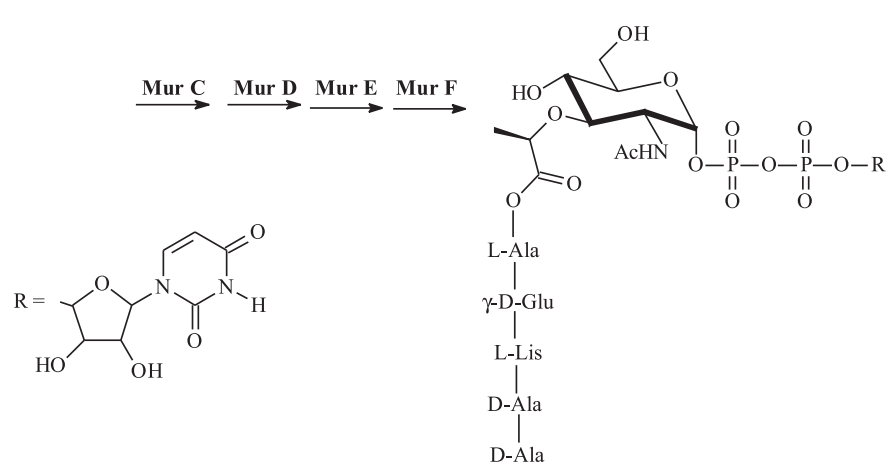

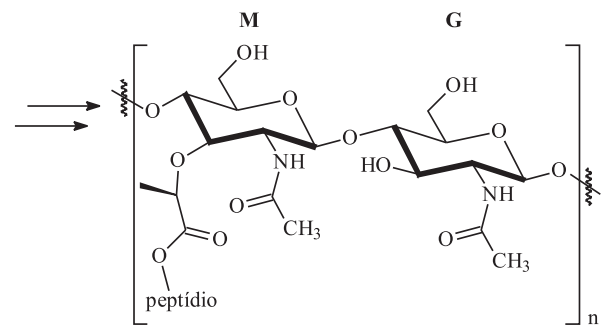

Peptidoglicano

Figura 1. Biossíntese do peptidoglicano 
éter do ácido uridina difosfato $N$-acetilmurâmico (UNAM) ${ }^{10}$, via transferência de dois elétrons através da NADPH ( $\beta$-nicotinamida adenina dinucleotídeo fosfato reduzido) utilizando como cofator o FAD (flavina adenina dinucleotídeo) ${ }^{8}$ (Figura 1).

Recentemente, 4-tiazolidinonas substituídas têm sido relatadas na literatura como potenciais novos agentes inibidores da enzima Mur $\mathrm{B}^{11}$, agindo como mimetizadores da porção difosfato da EP$\mathrm{UNAG}^{7}$. 4-tiazolidinonas representam uma classe de compostos de grande interesse científico devido às suas propriedades químicas e atividades biológicas, tais como antibacteriana ${ }^{12}$ (inclusive ativos contra Mycobacterium tuberculosis ${ }^{13}$ ), antifúngica ${ }^{14}$, antiprotozoária $^{15}$, anti-retroviral ${ }^{16}$, antiinflamatória e analgésica ${ }^{17}$, antitumoral ${ }^{18}$, dentre outras. A estrutura química base, conforme a IUPAC $^{19}$, desta classe de compostos derivados da tiazolidina, consiste em um anel de cinco membros contendo dois heteroátomos, um átomo de enxofre (posição 1) e um átomo de nitrogênio na posição 3 e um grupo carbonila na posição 4, podendo apresentar diversos grupos químicos em geral como substituintes nas posições 2, 3 e 5 (Figura 2).

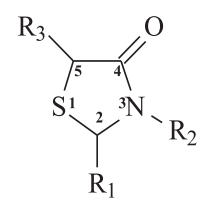

Figura 2. Anel 4-tiazolidinona

A síntese de compostos contendo o anel 4-tiazolidinona é bastante descrita na literatura ${ }^{7,9,20-22}$. Devido à busca por um método geral, simples, eficiente e de baixo custo para a obtenção destes compostos, vários trabalhos têm sido desenvolvidos ${ }^{23,26-32}$. Em geral, as 4-tiazolidinonas podem ser produzidas, com bons rendimentos, através de reações de ciclização envolvendo ácido $\alpha$-haloacético ou ácido $\alpha$-mercaptoacético, como descrito em revisões desenvolvidas por Brown ${ }^{24}$, em 1961, e Singh e colaboradores ${ }^{25}$, em 1981. Estas publicações são consideradas referências fundamentais para o estudo de 4-tiazolidinonas e por isso são freqüentemente citadas por diversos autores.

\section{MÉTODOS DE OBTENÇÃO DE 4-TIAZOLIDINONAS}

De acordo com a análise retrossintética visualizada para a síntese de derivados 4-tiazolidinônicos (Esquema 1), duas estratégias podem ser empregadas: formação das ligações $C_{5}-S$ e $C_{4}-N$, a partir de reações envolvendo compostos $\alpha$-aceto-halogenados e tioamidas (Esquema 1, 1) e formação das ligações $C_{2}-S$ e $C_{4}-N$ envolvendo reações entre iminas substituídas e ácido $\alpha$-mercaptoacético (Esquema 1, 2a) ou através de reações de condensação com três componentes, do tipo "one-pot", entre aminas, aldeídos e ácido $\alpha$ mercaptoacético (Esquema 1, 2b). Essa última estratégia conduz à formação de iminas in situ, a partir de reações entre aminas e

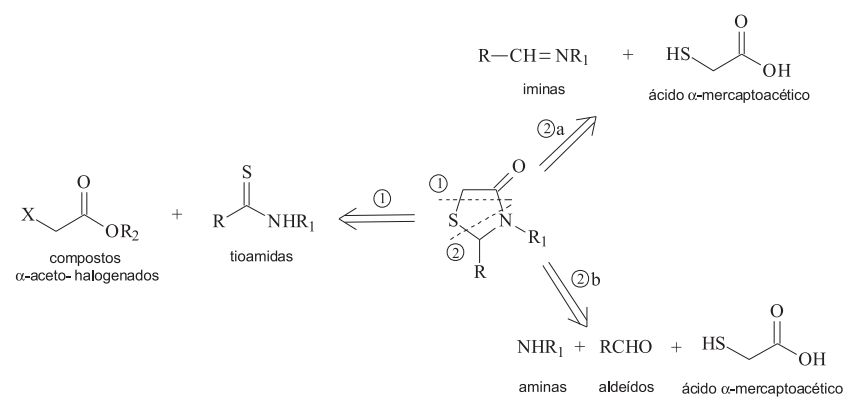

Esquema 1. Análise retrossintética de 4-tiazolidinonas aldeídos, e posterior condensação destas com o ácido $\alpha$-mercaptoacético, formando o anel 4-tiazolidinona.

Dentre os processos de síntese de 4-tiazolidinonas descritos na literatura, destacam-se as reações de ciclização envolvendo ácidos $\alpha$ haloacéticos ou derivados destes com tiouréias ${ }^{26}$, acil-tiossemicarbazidas $^{27}$ e tiossemicarbazonas ${ }^{28}$; reações de cicloadição do ácido $\alpha$-mercaptoacético com tiossemicarbazonas ${ }^{29}$; reações de condensação entre ácido $\alpha$-mercaptoacético, aminas primárias e aldeídos $^{30}$ e, reações de ciclização entre moléculas que apresentam ligação dupla ou tripla conjugada com o grupo carbonila, como anidrido maléico ou acetilenodicarboxilato de dimetila e compostos que apresentam a função tioamida, como as tiossemicarbazonas ${ }^{24,31-33}$.

\section{A partir de reações envolvendo ácidos $\alpha$-haloacéticos e seus derivados}

\section{Com tiouréias}

As reações de tiouréias substituídas com ácidos $\alpha$-haloacéticos, ou derivados destes, produzem 2-imino-4-tiazolidinonas. Estas reações ocorrem em solventes polares (ex.: etanol) sob refluxo e em presença de base (acetato de sódio anidro ou piridina) devido ao grupo imino ser muito susceptível à hidrólise ácida ${ }^{34}$. Tiouréias substituídas podem ser obtidas através de reações envolvendo fenilisotiocianatos com aminas primárias. Reações com tiouréias assimétricas (1,3 dissubstituídas, onde $\mathrm{R}_{1} \neq \mathrm{R}_{2}$ ) formam dois regioisômeros, onde a regiosseletividade é controlada por fatores eletrônicos, principalmente pela conjugação de substituintes elétron-atraentes (ex.: aril ou heteroaril) com o nitrogênio do grupo imino $^{34}$ (Esquema 2).
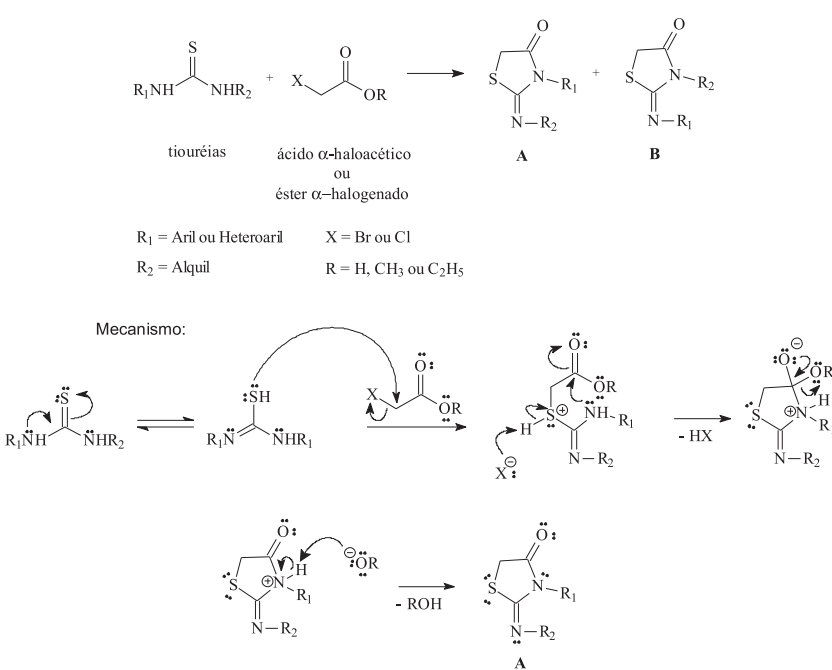

Esquema 2. Formação dos regioisômeros de 2-imino-4-tiazolidinonas

Ottanà e colaboradores ${ }^{34}$ demonstraram que a proporção entre os regioisômeros depende do solvente utilizado e do tempo de reação. Tendo como solvente $\mathrm{CHCl}_{3}$ e à temperatura ambiente, foi obtida após 4 h de reação, a razão isômero A/isômero B igual a 1/2, já em meio metanólico sob refluxo e após 24 h houve apenas a formação do isômero A. A proposta para explicar este fato é a existência de um rearranjo intramolecular convertendo o regioisômero B no regioisômero A (Esquema 3).

Reações envolvendo tiouréias assimétricas com substituintes 1,3dialquil, 1-alquil-3-aril ou 1-aril-3-alquil, possuem baixa regiosseletividade devido à falta de conjugação com o grupo imino. Laurent e colaboradores ${ }^{26}$ demonstraram que a reação de 1-benzil-3-(3clorobenzil)-tiouréia com bromoacetato de etila e dois equivalentes de $\mathrm{AcONa}$ em EtOH forma regioisômeros na proporção de 1:1. Os 


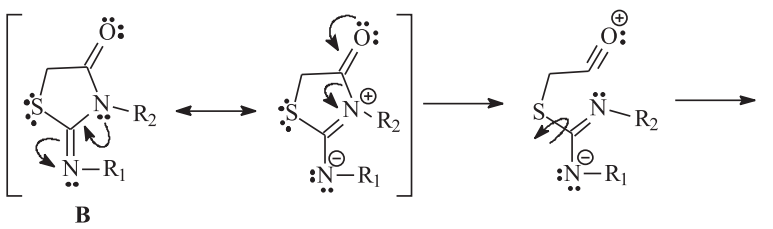

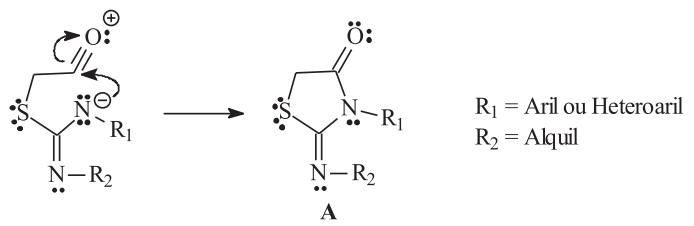

Esquema 3. Rearranjo intramolecular de 2-imino-4-tiazolidinonas

mesmos autores propuseram modificar um dos substituintes arilalquil para heteroarilalquil. O objetivo desta alteração foi avaliar uma possível regiosseletividade, devido à influência de uma possível ligação de hidrogênio entre o heterociclo protonado e a carbonila do éster, na formação do anel 4-tiazolidinona (Figura 3).<smiles>[R20]C1(OCC=[R])CSC(=NCC)N1</smiles>

Figura 3. Ligação de hidrogênio intramolecular determinando ciclização regiosseletiva

Para isso realizaram duas reações envolvendo a 1-etil-3-(2metilpiridinil)-tiouréia com bromoacetato de etila em EtOH, a primeira reação sem a adição de $\mathrm{AcONa}$, para facilitar a protonação do heterociclo, e a segunda com dois equivalentes de AcONa como descrito anteriormente. Foi observada uma proporção de 16:1 para os regioisômeros formados na primeira reação e de 1,4:1 para os obtidos na segunda reação (Esquema 4).

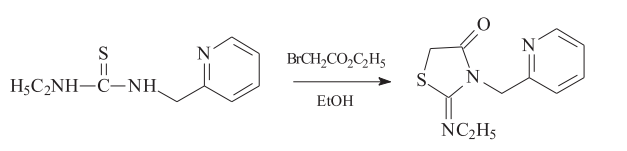

ausência de $\mathrm{AcON}$

2 eq. de $\mathrm{AcONa}$

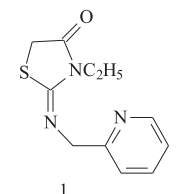

Esquema 4.Influência da utilização de base sobre a regiosseletividade

\section{Com aciltiossemicarbazidas e tiossemicarbazonas}

A preparação de 4-tiazolidinonas, a partir de reações envolvendo ácidos $\alpha$-haloacéticos ou seus derivados com tiossemicarbazonas e aciltiossemicarbazidas, é extensamente descrita na literatura ${ }^{31,35-37}$. Alves e colaboradores ${ }^{15}$ e Jolly e colaboradores ${ }^{38}$ sintetizaram 4-oxo- $\Delta^{2}$-tiazolin-2-il-hidrazonas, tautômeros de 4 tiazolidinonas, pela reação entre tiossemicarbazonas não substituídas em $N$-4 e ácidos monocloroacéticos substituídos, em presença de acetato de sódio anidro e etanol (Esquema 5).<smiles>[R]C1SC(NN=CC(=O)O)=NC1=O</smiles>

$\mathrm{R}=\mathrm{H}, \mathrm{CH}_{3}, \mathrm{CH}_{3} \mathrm{CH}_{2}$ ou $\mathrm{C}_{6} \mathrm{H}_{5}$

Esquema 5. Síntese do tautômero 4-oxo- $\Delta^{2}$-tiazolin-2-il-hidrazona
Metodologia semelhante foi aplicada em recentes trabalhos desenvolvidos por Küçükgüzel e colaboradores ${ }^{11,39}$ na obtenção de 2imino-4-tiazolidinonas a partir de reações envolvendo aciltiossemicarbazidas e $\alpha$-bromoacetato de etila (Esquema 6, Equação a).

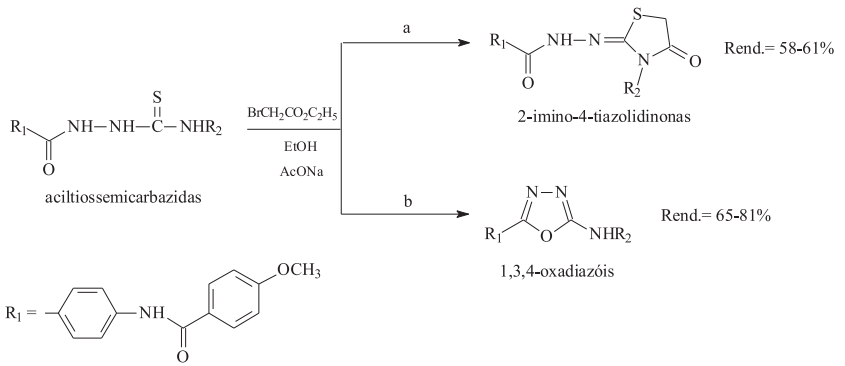

(a) $\mathrm{R}_{2}=\mathrm{CH}_{3}$ ou $\mathrm{CH}_{2} \mathrm{CH}_{2} \mathrm{CH}_{3}$

(b) $\mathrm{R}_{2}=\mathrm{C}_{6} \mathrm{H}_{5}$ ou $\mathrm{C}_{6} \mathrm{H}_{4} \mathrm{OCH}$

Esquema 6. Sintese de 4-tiazolidinonas e 1,3,4-oxadiazóis utilizando éster $\alpha$-halogenado

Contudo, esta metodologia não forneceu os derivados de 4tiazolidinonas esperados quando se tentou condensar as respectivas aciltiossemicarbazidas substituídas, contendo grupos volumosos em $N$-4, como fenil, $p$-metoxi-fenil e cicloexil, fornecendo por outro lado 1,3,4-oxadiazóis como principais produtos (Esquema 6, Equação b). Evidências da formação de 1,3,4-oxadiazóis foram comprovadas em espectros de RMN ${ }^{1} \mathrm{H}$ pela ausência do singleto de $\mathrm{S}-\mathrm{CH}_{2}$ localizado entre 3,95 e 4,09 ppm, mas presente em 4tiazolidinonas não substituídas na posição $2^{39}$.

O mecanismo proposto para formação de 4-tiazolidinonas ou de 1,3,4-oxadiazóis a partir de 1-acil-4-alquil/aril-tiossemicarbazidas envolve duas etapas: a primeira consiste em uma $S$-alquilação a partir da forma tiol e a segunda, na formação do heterociclo, com a perda de etanol, que é favorecida pela presença de um grupo pouco volumoso (ex.: metil) em $\mathrm{N}$-4, formando 4tiazolidinonas ou com a perda de mercaptoacetato de etila, que é favorecida pela presença de um grupo volumoso em $N-4$, formando 1,3,4-oxadiazóis ${ }^{11}$ (Esquema 7).

\section{A partir de reações envolvendo ácido $\alpha$-mercaptoacético}

\section{Com tiossemicarbazonas}

El-Gendy e colaboradores ${ }^{40}$ demonstraram que reações envolvendo ácido $\alpha$-mercaptoacético com tiossemicarbazonas, em benzeno seco, levam à formação de 3-tioureido-4-tiazolidinonas (Esquema 8).

\section{Com aminas e aldeídos}

Reações de condensação envolvendo ácido $\alpha$-mercaptoacético com aminas primárias ou ésteres de aminoácidos e aldeídos formando 4-tiazolidinonas 2,3-dissubstituídas são extensamente descritas na literatura ${ }^{41-46}$. Estas são denominadas reações de condensação de três componentes "one-pot", processos em que três substâncias são colocadas para reagirem diretamente em um único balão, facilitando a purificação e o isolamento do composto formado. Ocorrem geralmente em tolueno ou benzeno seco sob refluxo, com tempos de reação variando entre $2 \mathrm{~h}$ e $48 \mathrm{~h}$, alcançando bons rendimentos (Esquema 9).

Srivastava e colaboradores ${ }^{47}$ utilizaram outra metodologia, envolvendo reações de condensação de três componentes "one-pot", para obtenção de novas 4-tiazolidinonas. Foi utilizado como solvente o tetraidrofurano (THF) a $0{ }^{\circ} \mathrm{C}$ e dicicloexilcarbodiimida (DCC) 

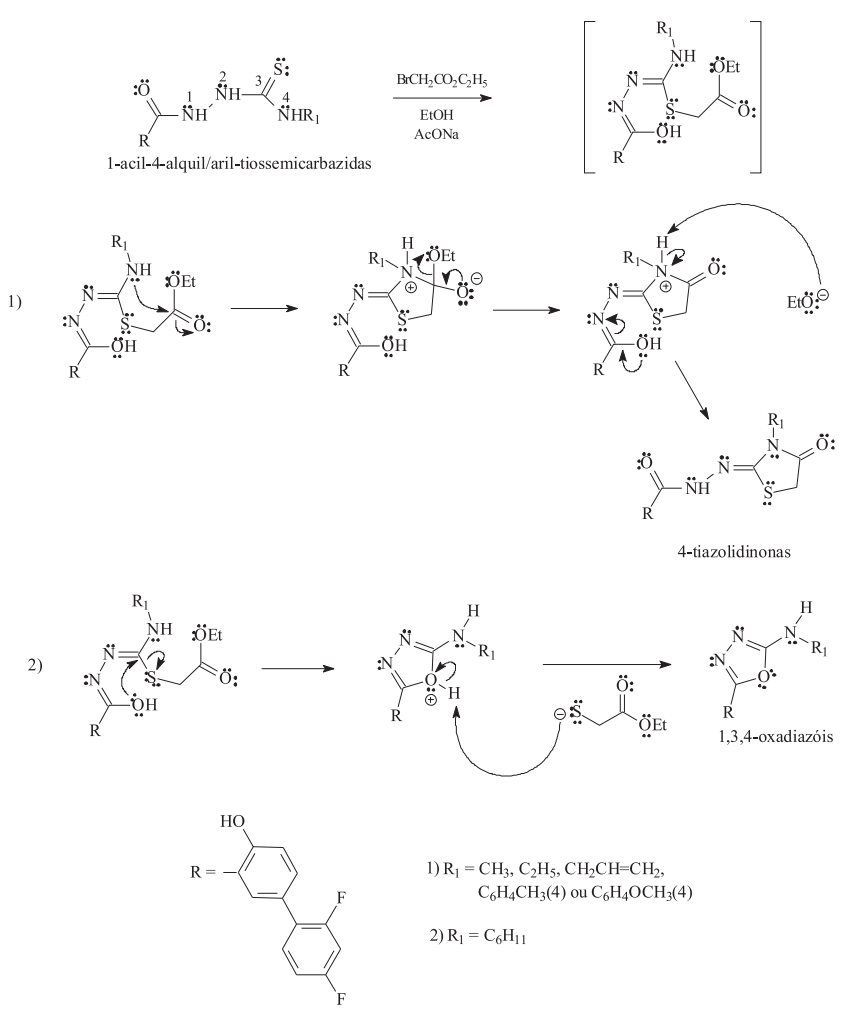

Esquema 7. Mecanismo de formação de 4-tiazolidinonas ou 1,3,4-oxadiazóis a partir de 1-acil-4-alqui-tiossemicarbazidas ou 1-acil-4-aril-tiossemicarbazidas

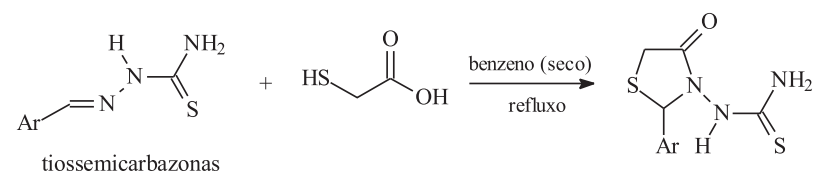

Esquema 8. Formação de 2-aril-3-tioureido-4-tiazolidinonas

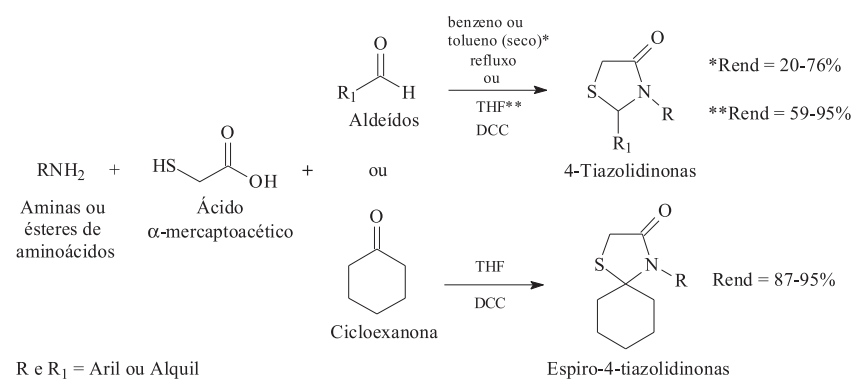

Esquema 9. Síntese de 4-tiazolidinonas utilizando reações de condensação de três componentes "one-pot"

como agente de acoplamento, reagente que atua diretamente na formação do anel tiazolidinônico (Esquema 9). Durante a reação a DCC é transformada em dicicloexiluréia (DCU) que pode ser facilmente removida por filtração, uma vez que a DCU é completamente insolúvel em THF. Os tempos médios de reação ficaram em torno de $1 \mathrm{~h}$, bem menores que aqueles observados nas reações realizadas em tolueno ou benzeno seco, atingindo bons rendimentos, principalmente quando a adição de DCC foi realizada a $0{ }^{\circ} \mathrm{C}$.

Em trabalho recente, Kavitha e colaboradores ${ }^{23}$ demonstraram que reações de ácido $\alpha$-mercaptoacético com aminas primárias e aldeídos ou cetonas podem ser otimizadas quando assistidas por microondas. Foi realizado um estudo comparativo entre o procedi- mento envolvendo DCC em THF e a reação assistida por microondas para a obtenção das mesmas 4-tiazolidinonas 2,3 dissubstituídas. Os rendimentos obtidos pelo método tradicional variaram entre 65 e 70\%, com tempos de reação no intervalo de 2 a 4 h, já os rendimentos das reações obtidas sob microondas variaram de 80 a $90 \%$, com tempos de reação na faixa de $45 \mathrm{~s}$ a 1 min. Os resultados obtidos podem ser interpretados como um aumento da regiosseletividade induzida por microondas. Este tipo de radiação atua promovendo a formação de estados de transição dipolares (ET1 e ET2), reduzindo a energia de ativação e, conseqüentemente, facilitando a formação do anel 4-tiazolidinona, como observado por Dandia e colaboradores ${ }^{20}$ para a síntese de espiro-4-tiazolidinonas a partir de isatinas, utilizando reações de condensação de três componentes "one-pot” (Esquema 10).

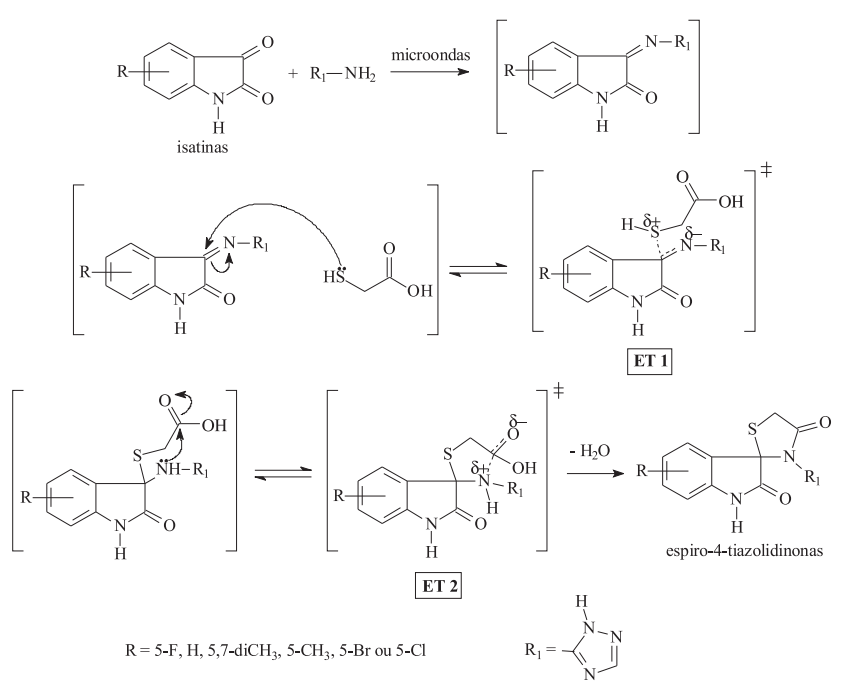

Esquema 10. Efeitos promovidos por microondas na formação de 4tiazolidinonas

A partir de reações entre compostos com a função tioamida e anidrido maléico ou acetilenodicarboxilato de dimetila

Outro método de preparação de 4-tiazolidinonas é através da reação envolvendo compostos que apresentam a função tioamida, como as tiossemicarbazonas ${ }^{33}$ com compostos carbonilados $\alpha, \beta$ insaturados, como acetilenodicarboxilato de dimetila (DMAD) $)^{31}$ ou anidrido maléico ${ }^{32}$. Berseneva e colaboradores ${ }^{31}$ sintetizaram $^{2}$ análogos 2,5-dimetileno-4-tiazolidinonas a partir da ciclização de derivados da malontioamida com DMAD (Esquema 11, Equação 1). Através da análise do espectro de $\mathrm{RMN}{ }^{13} \mathrm{C}$ foi possível observar a ausência de sinais entre 170-190 ppm, os quais são característicos de carbonos de tiocarbonilas e tiolactonas, um dos indícios que confirmaram a formação do anel 4-tiazolidinônico. Recentemente, Tenório e colaboradores ${ }^{32}$ obtiveram novos derivados, possuindo o grupo ácido acético em posição 5 do anel 4-tiazolidinona, a partir da reação entre nitrobenzaldeído-tiossemicarbazonas substituídas e anidrido maléico, em tolueno seco sob refluxo. Este processo consiste em uma reação de adição do tipo tia-Michael, estando o anidrido maléico no papel de aceptor de Michael (Esquema 11, Equação 2).

\section{REATIVIDADE DE 4-TIAZOLIDINONAS}

Dentre as diversas reações que envolvem o anel 4-tiazolidinona destacam-se os processos quimiosseletivos que ocorrem nas posições 1 (enxofre) em reações de oxidação ${ }^{48} ; 3$ (nitrogênio) em rea- 


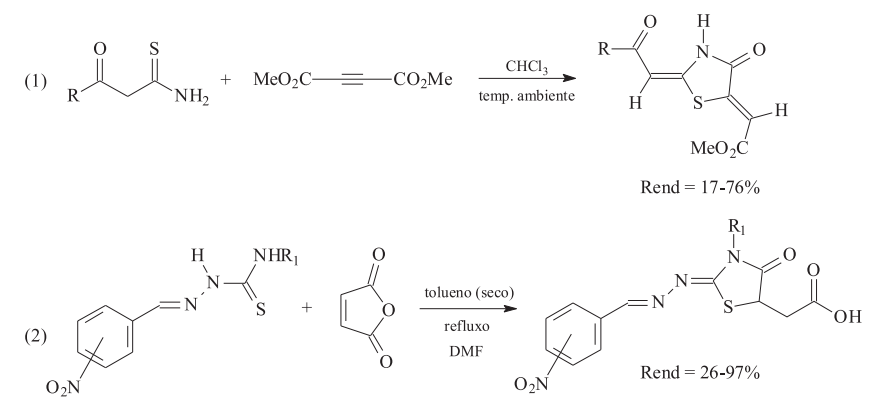

$$
\begin{aligned}
& \mathrm{R}=\mathrm{Et}, \mathrm{NMe}_{2}, \mathrm{NHC}_{6} \mathrm{H}_{4} \mathrm{Me}(2), \mathrm{NHC}_{6} \mathrm{H}_{3} \mathrm{Cl}_{2}(2,6), \mathrm{NHC}_{6} \mathrm{H}_{4} \mathrm{OMe}(2) \text {, 1-piperidinil ou 4-morfolinil } \\
& \mathrm{R}_{1}=\mathrm{H} \text {, Alquil ou Aril }
\end{aligned}
$$

Esquema 11. Obtenção de 4-tiazolidinonas a partir de compostos contendo a função tioamida

ções de $N$-alquilação ${ }^{49}$ e em reações de Mannich $^{25} ; 4$ (carbonila) em reações de tionação via reagente de Lawesson $^{50}$ e 5 (carbono metilênico) em reações de condensação com aldeídos ${ }^{2}$, cetonas ou com sais de diazônio ${ }^{24}$ (Figura 4).

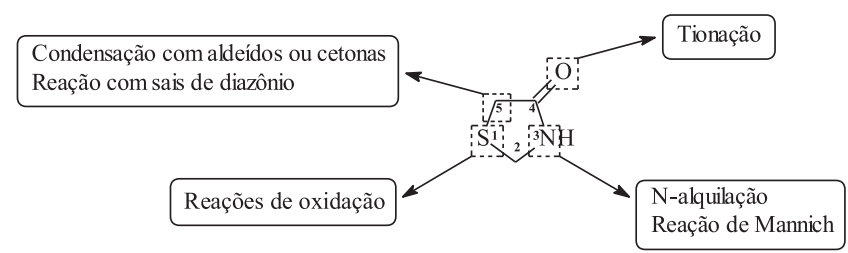

Figura 4. Principais reações do anel 4-tiazolidinona

\section{Reações de oxidação}

Reações de oxidação na posição 1 do anel 4-tiazolidinona são realizadas, geralmente, utilizando permanganato de potássio $\left(\mathrm{KMnO}_{4}\right)$ em ácido acético aquoso a $5{ }^{\circ} \mathrm{C}$, para a formação de 1,1-dioxo-4tiazolidinonas $^{48}$. Uma outra metodologia foi utilizada por Fuchigami e colaboradores ${ }^{51}$ utilizando o ácido $m$-cloro-perbenzóico (MCPBA), em clorofórmio, como agente oxidante (Esquema 12, Equação a).

\section{Reações de $N$-alquilação}

Trabalhos realizados por Graciet e colaboradores ${ }^{49}$ e Nishimoto e colaboradores $^{52}$ demonstraram que 4-tiazolidinonas substituídas na posição 2 com grupos alquil ou alquilalcóxi sofrem reações de $\mathrm{N}$ alquilação. Nesta síntese são utilizadas quantidades equivalentes de uma base, geralmente hidróxido de potássio, com 4-tiazolidinonas em dimetilformamida anidra, promovendo a formação do íon amideto através da abstração do hidrogênio em $N$-3 e posterior ataque deste ânion a 1,2-dibromoetano à temperatura ambiente (Esquema 12, Equação b). A utilização de uma base dura como o hidróxido de potássio é imprescindível para a formação do ânion amideto, uma vez que 4tiazolidinonas não substituídas na posição 3 são ácidos fracos.

\section{Reação de Mannich}

Reações do tipo Mannich ocorrem geralmente entre 2-imino4-tiazolidinonas e aminas primárias ou secundárias, em presença de formaldeído $(\mathrm{HCHO})$ ou paraformaldeído [(HCHO) $\left.{ }_{\mathrm{n}}\right]$, em metanol, levando à formação de 2-imino-3-(aminometil substituído)-4-tiazolidinona ${ }^{25}$ (Esquema 12, Equação c).
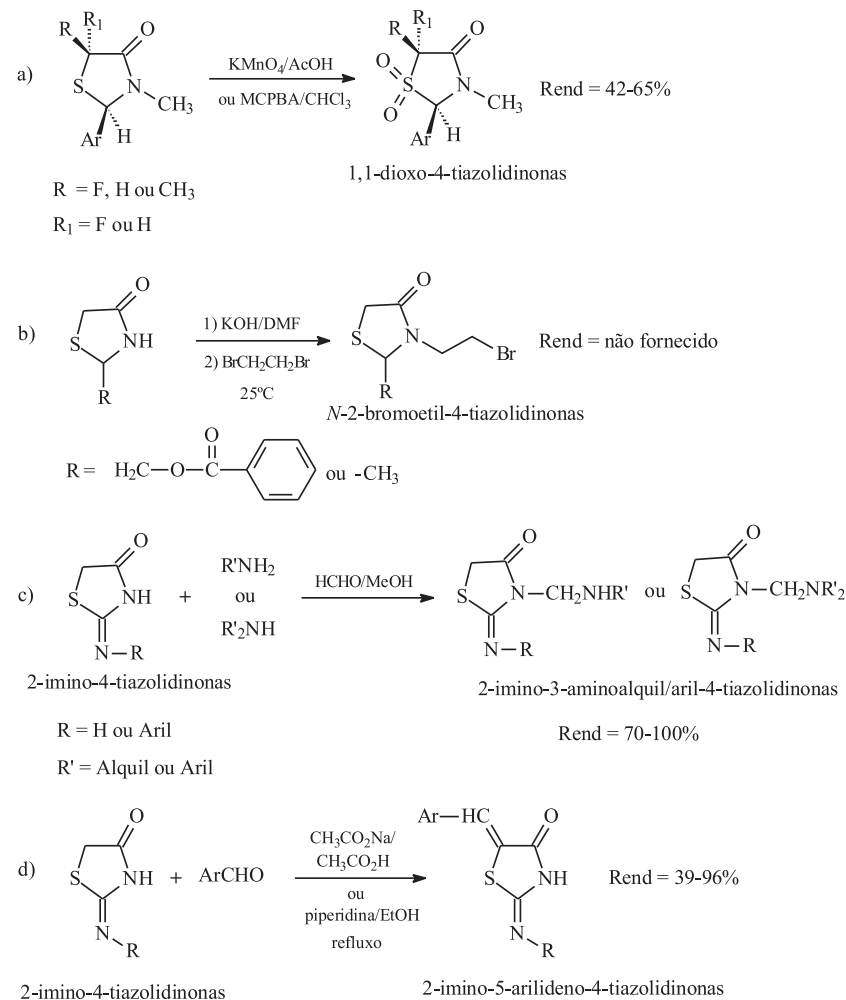

2-imino-4-tiazolidinonas

2-imino-5-arilideno-4-tiazolidinonas

$\mathrm{R}=$ Heterociclo ou Aril<smiles>[R][R4][Y4]([H])=C1SC(N=N[Al])C(=O)N1[R]</smiles>

$\mathrm{R}=$ Aril, Alquil ou Heterociclo

$\mathrm{X}=\mathrm{O}, \mathrm{S}$ ou $\mathrm{NR}$

$\mathrm{O}=2,4$-tiazolidinadionas

$\mathrm{X}\{\mathrm{S}=2$-tioxo-4-tiazolidinonas

$\mathrm{NR}=2$-imino-4-tiazolidinonas

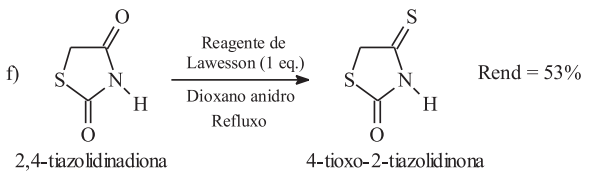

2,4-tiazolidinadiona $\quad$-tioxo-2-tiazolidinona

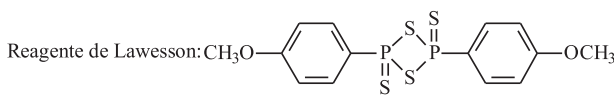

Esquema 12. Principais reações de compostos contendo o anel 4tiazolidinônico

\section{Reações de condensação com aldeídos}

O grupo metileno na posição 5 do anel 4-tiazolidinona, devido à sua acidez, possui reatividade característica, e condensa com aldeídos ou cetonas em reação do tipo Knoevenagel ${ }^{53}$. Nesta reação há formação de um enolato intermediário estabilizado, que é dependente do efeito elétron-atraente da carbonila, adjacente ao grupo metileno, e da presença de grupos elétron-atraentes na posição 2 do anel 4-tiazolidinona ${ }^{25}$. A reação de condensação ocorre geralmente em presença de solução tamponada ácido acético glacial/acetato de sódio anidro ${ }^{2}$, onde o acetato de sódio funciona tanto como base quanto como agente desidratante, ou alternativamente em solução piperidina/etanol ${ }^{34}$ (Esquema 12, Equação d).

A síntese de 5-arilidenos-4-tiazolidinonas, obtidos pela condensação de 4-tiazolidinonas com benzaldeídos substituídos, conduz, teoricamente, à duas configurações diastereoisoméricas $E$ 
e Z. A configuração na qual os dois substituintes prioritários (enxofre e fenil) se encontram do mesmo lado é denominada $Z$; quando estes substituintes estão localizados em lados opostos é denominada $E$. A determinação da configuração dos diastereoisômeros pode ser feita através de análises espectroscópicas ( $\mathrm{RMN}{ }^{13} \mathrm{C}, \mathrm{RMN}$ $\left.{ }^{1} \mathrm{H}\right)$ e através de cristalografia por raios X. Otanná e colaboradores $^{34}$ determinaram a configuração $Z$ para análogos 2-imino-5arilideno-4-tiazolidinonas verificando a influência magnética da carbonila sobre o deslocamento químico do hidrogênio metínico em $\mathrm{RMN}{ }^{1} \mathrm{H}$, que aparece como um singleto em 7,70-7,75 ppm, enquanto que para isômeros $E$, devido à menor influência magnética do átomo de enxofre, este singleto aparece com deslocamento inferior a $7,70 \mathrm{ppm}$.

\section{Reações com sais de diazônio}

4-tiazolidinonas podem realizar reações de acoplamento com sais de diazônio ${ }^{24}$. Estas reações, assim como as reações de condensação aldólica já descritas, são realizadas via formação de enolato (Esquema 12, Equação e).

\section{Reações de tionação}

A tionação de 4-tiazolidinonas pode ser realizada utilizando o reagente de Lawesson (1,3-ditio-2,4-difosfatano-2,4-dissulfeto-2,4bis(4-metóxifenil)), como descrito por Andrade e colaboradores ${ }^{50}$, para a síntese de 4-tioxotiazolidin-2-ona a partir de tiazolidina2,4-diona, em dioxano anidro. (Esquema 12, Equação f).

A velocidade desta reação é dependente da densidade eletrônica existente na carbonila e limitada pela temperatura ${ }^{54}$. Em compostos contendo duas ou mais carbonilas, sofrerá primeiramente tionação aquela que for mais rica em elétrons. A conversão do grupo carbonila em tiocarbonila é atribuída a ilídeos de ditiofosfina $\left(p-\mathrm{CH}_{3} \mathrm{O}-\mathrm{C}_{6} \mathrm{H}_{4}-\mathrm{PS}_{2}\right)$, formados a partir da quebra do anel de quatro membros, contendo átomos de fósforo e enxofre alternados, existente no reagente de Lawesson ${ }^{55}$. O mecanismo reacional envolve um intermediário de quatro membros semelhante à oxafosfetana, observada na reação de Wittig, para formação de alquenos a partir de aldeídos e cetonas ${ }^{55,56}$.

\section{IMPORTÂNCIA BIOLÓGICA DE 4-TIAZOLIDINONAS}

$\mathrm{O}$ anel 4-tiazolidinona per si não apresenta atividade biológica. No entanto, compostos contendo esse heterociclo possuem grande interesse científico, pois diversos trabalhos relatam um amplo espectro de atividades biológicas dos seus diferentes deriva$\operatorname{dos}^{7,11,31,57,58}$. Como visto anteriormente, o anel 4-tiazolidinona possui vários sítios de substituição, o que leva a um grande número de análogos estruturais. Portanto, as diferentes atividades biológicas podem ser atribuídas a grupos substituintes nas posições 2,3 e 5 do anel, onde promovem modificações nos parâmetros físico-químicos e estruturais (lipofílicos, eletrônicos, polares e estéricos) das moléculas. Serão descritas, a seguir, as principais atividades biológicas apresentadas por esses compostos.

\section{Atividade antimicrobiana}

Andres e colaboradores ${ }^{7}$ demonstraram uma potencial inibição da enzima Mur B por 4-tiazolidinonas 2,3,5-trissubstituídas, contendo um grupo ácido acético $\alpha$-substituído em $N$-3. Foi observado que o derivado não substituído na posição 5 do anel 4-tiazolidinona e contendo o radical $n$-butil em $\alpha$ ao grupo ácido acético (sintetizado a partir da $D$-norleucina) possuía maior poder inibitório sobre a enzima Mur B $\left(\mathrm{IC}_{50}=7,7 \mu \mathrm{M}\right)$ (Figura 4a). Segundo os autores, a estereoquímica parece desempenhar um papel significante na potência da atividade antibacteriana, uma vez que o derivado obtido a partir da $L$-norleucina mostrou-se quatro vezes menos ativo $\left(\mathrm{IC}_{50}=\right.$ $28,4 \mu \mathrm{M}$ ). Essas constatações foram realizadas utilizando-se dados obtidos a partir de testes in vitro, $\mathrm{IC}_{50}$ (concentração em que $50 \%$ da atividade enzimática é reduzida) e recursos de química computacional, como docagem de substratos, tendo como base os dados de cristalografia por raios X da enzima Mur B (Figura 5a). Recentemente, Küçükgüzel e colaboradores ${ }^{11}$ realizaram a avaliação antimicrobiana de 4-tiazolidinonas, derivadas de hidrazidashidrazonas do ácido 2',4'-difluoro-4-hidroxibifenil-3-carboxílico (diflunizal), frente a 56 espécies de bactérias e 6 de fungos, onde não foi observada nenhuma atividade anti-microbiana significante. Por outro lado, Kavitha e colaboradores ${ }^{23}$ relataram que análogos 4-tiazolidinonas substituídas em $N$-3, com um grupo derivado da venlafaxina (1-[2-amino-1-(4-metoxi-fenil)-etil]-cicloexanol), possuíam atividade frente a várias cepas bacterianas, como Pseudomonas fluorescens, e de fungos, como espécies de Trichoderma. Alguns dos resultados obtidos, medindo-se o diâmetro do halo de inibição e determinando-se a concentração inibitória mínima (CIM), mostraram uma melhor ação antimicrobiana destes análogos que os fármacos padrões utilizados, estreptomicina (antibiótico) e nistatina (antifúngico) (Figura 5b).

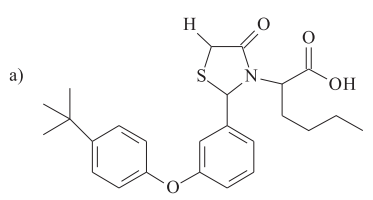

$\mathrm{R}=\mathrm{H}, \mathrm{CH}_{3}$ ou $\mathrm{C}(\mathrm{O}) \mathrm{N}(\mathrm{H}) \mathrm{NHPh}$ b)

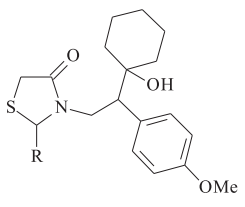

$\mathrm{R}=$ Aril ou Heterociclo
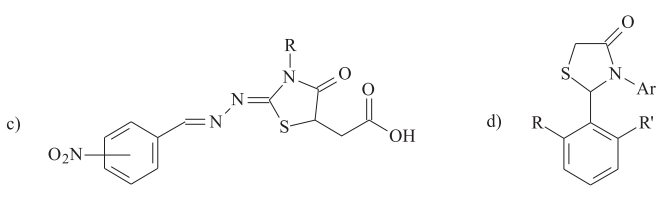

$\mathrm{R}=\mathrm{F}$ ou $\mathrm{Cl} \mathrm{R}^{\prime}=\mathrm{F}$ ou $\mathrm{Cl}$
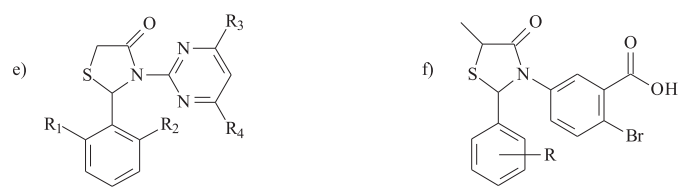

$\mathrm{R}_{1}=\mathrm{F}$ ou $\mathrm{Cl} \quad \mathrm{R}_{2}=\mathrm{F}$ ou Cl

$\mathrm{R}_{3}=\mathrm{H}, \mathrm{Cl}, \mathrm{CH}_{3}, \mathrm{OCH}_{3}$ ou OH

$\mathrm{R}=\mathrm{H}, 2-\mathrm{OH}, 2-\mathrm{OCH}_{3}$ ou $2-\mathrm{OCH}_{3}$

$\mathrm{R}_{4}=\mathrm{H}$ ou $\mathrm{CH}_{3}$<smiles>[R]C1SCC(=O)N1CCN1C(=O)CSC1[R]</smiles>

h)

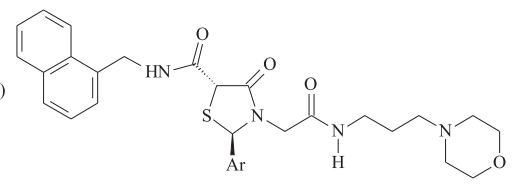

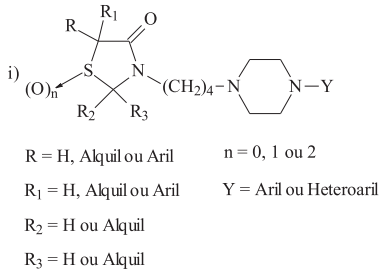

Figura 5. Estruturas de algumas 4-tiazolidinonas de importância biológica 


\section{Atividade antiprotozoária}

Alves e colaboradores ${ }^{15}$ realizaram avaliação da atividade antiprotozoária, in vitro, para análogos 4-oxo- $\Delta^{2}$-tiazolin-2-ilhidrazonas frente à Entamoeba histolytica e Trichomonas vaginalis. Foi observada uma menor atividade antiprotozoária dos compostos testados $(\mathrm{CIM}=10-100 \mu \mathrm{g} / \mathrm{mL})$ que o metronidazol $(\mathrm{CIM}=$ $1,25-2,5 \mu \mathrm{g} / \mathrm{mL}$ ), fármaco utilizado como referência. Recentemente, Tenório e colaboradores ${ }^{31}$ relataram uma excepcional atividade anti-Toxoplama gondii, in vitro, de derivados do ácido 2[(fenilmetileno)hidrazono]-3-fenil-4-oxo-5-tiazolidinoacético (Figura 5c). Todos os compostos analisados mostraram ser menos tóxicos e mais eficazes contra o parasita intracelular que a hidróxiuréia (substância com ação comprovada frente à $T$. gondii), e alguns destes apresentaram melhores atividades antiprotozoárias que a sulfadiazina, fármaco utilizado como referência.

\section{Atividade antiviral}

Recentes trabalhos relatam uma potencial inibição da enzima transcriptase reversa do vírus da imunodeficiência humana tipo I (RTHIV-1), promovida por compostos não-nucleosídeos contendo o anel 4-tiazolidinona. Barreca e colaboradores ${ }^{41-43}$ demonstraram que 4tiazolidinonas 2,3-diaril-substituídas contendo em C-2 grupos 2',6'di-halofenil (Figura 5d) possuem uma maior ação inibitória seletiva frente a RT-HIV-1 e menor citotoxicidade, in vitro, em células MT-4 infectadas, que os derivados 1 -aril- $1 H, 3 H$-tiazolo[3,4- $\alpha]$ benzimidazóis (TBZs), potentes agentes supressores não nucleosídeos da RT-HIV-1, utilizados como referência. Estudo realizado por Rawal e colaboradores ${ }^{15}$ utilizando derivados 2-aril-3-furfuril-4-tiazolidinonas indicou que apenas os compostos contendo, em C-2, grupos 2',6'-di-halofenil possuem ação inibitória relevante sobre a RT-HIV-1. Rao e colaboradores ${ }^{21}$ realizaram estudo semelhante com análogos 2-(2,6-di-halofenil)3-(pirimidin-2-il)-1,3-tiazolidin-4-onas obtiveram elevados níveis de atividade inibitória sobre a RT-HIV-1 (Figura 5e). De fato, a presença de halogênios nas posições 2 e 6 restringe a rotação do núcleo fenila, permitindo a formação de uma conformação do tipo "borboleta" existente em vários inibidores não-nucleosídeos da transcriptase reversa do HIV-1 conhecidos ${ }^{58}$.

\section{Atividades antiinflamatória e analgésica}

Com a descoberta e caracterização da enzima cicloxigenase tipo 2 (COX-2) no final da década de 90, ocorreu um melhor entendimento de como funciona o processo inflamatório e, conseqüentemente, um aumento no interesse da produção de novas substâncias inibidoras seletivas $\mathrm{COX}-2^{59}$, isentas dos efeitos gastro-intestinais atribuídos à inibição da COX-1. As atividades antiinflamatória e analgésica têm sido descritas em diversos trabalhos recentemente desenvolvidos para 4-tiazolidinonas. Goel e colaboradores ${ }^{60}$ obtiveram excepcional resultado para uma das 3-(4-bromo-2-carbo-xifenil)5-metil-4-tiazolidinonas 2-substituídas testadas sobre a redução de edema (induzido por carragenina) em ratos albinos, utilizando como fármaco padrão a fenilbutazona (Figura 5f). Em 1991, Walsh e colaboradores $^{61}$ descreveram a ação antiinflamatória e patentearam processos de síntese envolvendo mais de 80 derivados contendo o heterociclo 4-tiazolidinona, relatando a atuação destes compostos como inibidores das enzimas cicloxigenase e 5-lipoxigenase.

Vigorita e colaboradores ${ }^{59}$ realizaram estudo computacional e, posteriormente, avaliação in vitro da ação inibitória seletiva das enzimas COX-1 e COX-2 promovida por estereoisômeros de 3,3'(1,2-etanodiil) bis[2-(3,4-dimetóxifenil)-4-tiazolidinonas (Figura $5 \mathrm{~g})$. Estas moléculas possuem dois centros estereogênicos $(C-2 \mathrm{e}$
$C-2$ '), sendo objeto deste estudo os isômeros $R R, S S$, o racemato $(R R+\mathrm{SS})$ e o composto meso $(R S)$, onde foi observado, em ensaios in vitro, que o composto dextrorrotatório possuía atividade seletiva COX-2 semelhante ao rofecoxib (fármaco-referência utilizado). Através de modelagem molecular foi possível determinar a ordem de seletividade para a COX-2: $S S^{\prime}>R S>R R^{\prime}$. Allen e colaboradores ${ }^{62}$ desenvolveram novas 4-tiazolidinonas 2,3,5-trissubstituídas com potencial ação antagonista de receptores de quemoquinas CCR4, os quais são responsáveis pela migração de células $\mathrm{T}$ ao local da inflamação, que desempenham papel principal na condução de processos inflamatórios alérgicos (Figura 5h).

\section{Atividade antipsicótica}

Hrib e colaboradores ${ }^{63}$ patentearam um processo de síntese envolvendo 4-(3-(4-oxotiazolidinil)butinilaminas-4-tiazolidinonas (Figura 5i) e 3-[4(4-piperazinil-1-il)butil]-4-tiazolidinonas (Figura 5j), relatando a ação dos primeiros compostos como analgésicos e antihipertensivos, e dos segundos como analgésicos, ansiolíticos, anticonvulsivantes e antipsicóticos. A ação antipsicótica é determinada pela afinidade destes compostos por receptores $5 \mathrm{HT}_{2}$ (serotonina) e $\mathrm{D}_{2}$ (dopamina).

\section{Atividade antagonista sobre o fator ativador de plaquetas (FAP)}

Enomoto e colaboradores ${ }^{64}$ patentearam um processo de síntese envolvendo novos derivados 2-(3-piridil)-4-tiazolidinonas, e sua aplicação como antagonista seletivo do fator ativador de plaquetas (FAP). Concentrações extremamente baixas deste fator são suficientes para produzir diversos efeitos fisiológicos, tais como hipotensão sanguínea, aumento da permeabilidade vascular, contração do músculo liso, ativação de leucócitos, monócitos e macrófagos e aceleração da decomposição do glicogênio hepático. É atribuído a estes efeitos uma série de implicações, dentre as quais destacam-se diversos tipos de inflamações, doenças alérgicas, circulatórias e gastrointestinais.

\section{CONCLUSÕES}

Compostos contendo o anel 4-tiazolidinona vêm, nos últimos anos, ganhando importância junto à comunidade científica. Vários trabalhos foram e estão sendo desenvolvidos por diversos grupos de pesquisa, tanto da academia quanto das indústrias farmacêuticas (ou mesmo em trabalho conjunto), com base nesta classe de compostos. Sob o ponto de vista químico, o interesse por estas moléculas deve-se ao baixo custo e à versatilidade de sua síntese, utilizando geralmente ácido $\alpha$-cloroacético, ácido $\alpha$-mercaptoacético ou seus derivados, obtendo-se análogos com grande diversidade estrutural; e da quimiosseletividade envolvendo reações com este heterociclo, podendo o mesmo sofrer reações de $N$ alquilação, oxidação e reações envolvendo o caráter nucleofílico do carbono metilênico, como em reações de condensação com aldeídos aromáticos e sais de diazônio. Sob o ponto de vista biológico, as 4-tiazolidinonas apresentam diversas atividades, sendo relatadas como potenciais agentes antimicrobianos, anti-retrovirais, antiinflamatórios, anti-hipertensivos, antipsicóticos, anticoagulantes, antiprotozoários etc. Com isso, vislumbra-se, a partir desta classe de substâncias, o surgimento de novos fármacos.

\section{AGRADECIMENTOS}

À Coordenação de Aperfeiçoamento de Pessoal de Nível Superior (CAPES) e ao Conselho Nacional para o Desenvolvimento Científico e Tecnológico $(\mathrm{CNPq})$. 


\section{REFERÊNCIAS}

1. Kollef, M. H.; Micek, S. T.; Curr. Opin. Infect. Dis. 2006, 19, 161.

2. Vicini, P.; Geronikaki, A.; Anastásia, K.; Incerti, M.; Zani, F.; Bioorg. Med. Chem. 2006, 14, 3859.

3. Tenover, F. C.; McDonald, L. C.; Curr. Opin. Infect. Dis. 2005,18, 300

4. Ellis, M. W.; Lewis, J. S.; Curr. Opin. Infect. Dis. 2005,18, 496.

5. Pfeltz, R. F.; Wilkinson, B. J.; Curr. Drug Targets Infect. Disord. 2004, 4, 273.

6. Khan, M. W.; Alam, M. J.; Rashid, M. A.; Chowdhury, R.; Bioorg. Med. Chem. 2005, 13, 4796.

7. Andres, C. J.; Bronson, J. J.; D'Andrea, S. V.; Deshpande, M. S.; Falk, P. J.; Grant-Young, K. A.; Harte, W. E.; Ho, H. T.;Misco, P. F.; Robertson, J. G.; Stock, D.; Sun, Y.; Walsh, A. W.; Bioorg. Med. Chem. Lett. 2000,10, 715.

8. Benson, T. E.; Walsh, C. T.; Massey, V.; Biochemistry 1997, 36, 796.

9. Park, J. T. Em Escherichia coli and Salmonella typhimurium: Cellular and Molecular Biology; Neidhardt, F. C.; Ingraham, J. L.; Low, K. B.; Magasanik, B.; Schaechter, M.; Umbarger, H. E., eds., American Society for Microbiology: Washington, DC, 1987, p. 663-671,

10. Bugg, T. D. H.; Walsh, C. T.; Nat. Prod. Rep. 1993, 199.

11. Küçükgüzel, G.; Kocatepe, A.; De Clercq, E.; Sahin F.; Güllüce, M.; Eur. J. Med. Chem. 2006, 41, 353.

12. Khawass, S. M. E.; Khalil, M. A.; Chaaban, I.; Il Farmaco 1989, 44, 415.

13. Babaoglu, K.; Page, M. A.; Jones, V. C.; McNeil, M. R.; Dong, C.; Naismith, J. H.; Lee, R. E.; Bioorg. Med. Chem. Lett. 2003, 13, 3227.

14. Capan, G.; Ulusoy, N.; Ergenc, N.; Kiraz, M.; Monatsh. Chem. 1999, 130, 1399.

15. Alves, A. J.; Leite, A. C. L.; Santana, D. P.; Beltrão, T. M.; Coelho, M. R. D.; Il Fármaco 1993, 48, 1167.

16. Rawal, R. K.; Prabhakar, Y. S.; Katti, S. B.; De Clercq, E.; Bioorg. Med. Chem. 2005,13, 6771.

17. Vigorita, M. G.; Ottanà, R.; Monforte, F.; Maccari, R.; Trovato, A.; Monforte, M. T.; Taviano, M. F.; Bioorg. Med. Chem. Lett. 2001, 11, 2791

18. Gududuru, V.; Hurh, E.; Dalton, T. J.; Miller, D. D.; J. Med. Chem. 2005, 48,2584

19. Panico, R.; Powell, W. H.; Richer, J. C., eds.; IUPAC - Nomenclature of Organic Compounds, Blackwell: London, 1993.

20. Dandia, A.; Singh, R.; Khaturia, S.; Mérienne, C.; Morgantc, G.; Loupyd, A.; Bioorg. Med. Chem. 2006, 14, 2409.

21. Rao, A.; Balzarini, J.; Carbone, A.; Chimirri, A.; De Clercq, E.; Monforte, A. M.; Monforte, P.; Pannecouque, C.; Zappalà, M.; Antiviral Res. 2004, 63,79 .

22. Altintaș, H.; Ateș, Ö.; Birteksöz, S.; Ötük, G.; Uzun, M.; Şatana, D.; Turk. J. Chem. 2005, 29, 425.

23. Kavitha, C. V.; Basappa; Nanjunda, S. S.; Mantelingu, K.; Doreswamy, S.; Sridhar, M. A.; Prasad, J. S.; Rangappa, K. S.; Bioorg. Med. Chem. 2006, 14, 2290.

24. Brown, F. C.; Chem. Rev. 1961, 61, 463

25. Singh, S. P.; Parmar, S. S.; Raman, K.; Chem. Rev. 1981, 81, 175.

26. St. Laurent, D R.; Dedong - Wu, Q. G.; Serrano-Wu, M. H.; Tetrahedron Lett. 2004, 45, 1907.

27. Bonde, C. G.; Gaikwad, N. J.; Bioorg. Med. Chem. 2004, 12, 2151

28. Ergenç, N.; Çapan, G.; Günay, N.S.; Özk1r1ml1, S.; Güngör, M.; Özbey, S.; Kendi, E.; Arch. Pharm. Med. Chem. 1999, 332, 343

29. Yadav, L. D. S.; Vaish, A.; Sharma, S.; J. Agric. Food Chem. 1994, 42, 881

30. Holmes, C. P.; Chinn, J. P.; Gordon, E. M.; Gallop, M. A.; J. Org. Chem. 1995, 60, 7328 .

31. Berseneva, V. S.; Tkachev, A. V.; Morzherin, Y. Y.; Dehaen, W.; Luyten, I.; Toppet, S.; Bakulev, V. A.; J. Chem. Soc., Perkin Trans. 1 1998, 2133.

32. Tenório, R. P.; Carvalho, C. S.; Pessanha, C. S.; Lima, J. G.; de Faria, A. R.; Alves, A. J.; Melo, E. J. T.; Góes, A. J. S.; Bioorg. Med. Chem. Lett. 2005, 15, 2575.

33. Aquino, T. M.; Liesen, A. P.; Lima, J. G.; Silva, R. E. A.; Lima, V. T.; Araújo, J. M.; Goés, A. J.; Resumos da $29^{a}$ Reunião Anual da Sociedade Brasileira de Química, Águas de Lindóia, Brasil, 2006.
34. Ottanà, R.; Maccari, R.; Barreca, M. L.; Bruno, G.; Rotondo, A.; Rossi, A.; Chiricosta, G.; Di Paola, R.; Sautebin, L.; Cuzzocrea, S.; Vigorita, M. G.; Bioorg. Med. Chem. 2005, 13, 4243.

35. Meher, S. S.; Naik, S.; Behera, R. K.; Nayak, A.; J. Indian Chem. Soc. 1981, 58, 274.

36. Gürsoy, A.; Terzioglu, N.; Ötuk, G.; Eur. J. Med. Chem. 1997, 32, 753.

37. Abbady, M. A.; Hafez, S. H. A.; Kandeel, M. M.; Monem, M. I. A.; Molecules 2003, 8,622 .

38. Jolly, V. S.; Sharma, K. P.; J. Indian Chem. Soc. 1990, 67, 412.

39. Kucukguzel, S. G.; Oruc, E. E.; Rollas, S.; Sahin, F.; Ozbek, A.; Eur. J. Med. Chem. 2002, 37, 197.

40. El-Gendy, Z.; Abdel-Rahman, R. M.; Fawzy, M. M.; J. Indian Chem. Soc. 1990, 67, 927.

41. Barreca, M. L.; Chimirri, A.; De Luca, L.; Monforte, A. M.; Monforte, P.; Rao, A.; Zappalà, M.; Balzarini, J.; De Clercq, E.; Pannecouque, C.; Witvrouw, M.; Bioorg. Med. Chem. Lett. 2001, 11, 1793.

42. Barreca, M. L.; Balzarini, J.; Chimirri, A.; Clercq, E. D.; Luca, L. D.; Holtje, H. D.; Holtje, M.; Monforte, A. M.; Monforte, P.; Pannecouque, C.; Rao, A.; Zappala, M.; J. Med. Chem. 2002, 45, 5410.

43. Barreca, M. L.; Chimirri, A.; De Clercq, E.; De Luca, L.; Monforte, A. M.; Monforte, P.; Rao, A.; Zappalà, M.; Il Farmaco 2003, 58, 259.

44. Rao, A.; Balzarini, J.; Carbone, A.; Chimirri, A.; De Clercq, E.; Monforte, A. M.; Monforte, P.; Pannecouque, C.; Zappalà, M.; Antiviral Res. 2004, 63, 79.

45. Diurno, M. V.; Mazzoni, O.; Piscopo, E.; Calignano, A.; Giordano, F.; Bolognese, A.; J. Med. Chem. 1992, 35, 2910.

46. Gududuru, V.; Hurh, E.; Dalton, J. T.; Miller, D. D.; Bioorg. Med. Chem. Lett. 2004, 14, 5289

47. Srivastava, T.; Haq, W.; Katti, S. B.; Tetrahedron 2002, 58, 7619

48. Johnson, M. R.; Fazio, M. J.; Ward, D. L.; Sousa, L. R.; J. Org. Chem. 1983, 48, 494

49. Graciet, J. C.; Niddam, V.; Gamberoni, M.; Trabaud, C.; Dessolin, J.; Medou, M.; Mourier, N.; Zoulim, F.; Bore, 1. C.; Hantz, O.; Camplo, M.; Chermann, J. C.; Kraus, J. L.; Bioorg. Med. Chem. Lett. 1996, 6, 1775

50. Andrade, A. M. C.; Lima, W. T.; Rocha, M. P. A.; Lima, M. C. A.; Galdino, S. L.; Barbosa Filho, J. M.; Góes, A. J. S.; Pitta, I. R.; Boll. Chim. Farmac. 2002, 141, 428

51. Fuschigami, T.; Narizuka, S.; Konno, A.; J. Org. Chem. 1992, 57, 3755.

52. Nishimoto, S. I.; Hatta, H.; Ueshima, H.; Kagiya, T. L.; J. Med. Chem. 1992, 35, 2712

53. Subba Rao, Y. V.; Choudary, B. M.; Synth. Commun. 1991, 21, 1163

54. Clausen, K.; Thorsen, M.; Lawesson, S. O.; Tetrahedron 1981, 37, 3635.

55. Cava, M.P.; Levinson, M. I.; Tetrahedron 1985, 41, 5061.

56. Souza, M. V.; Ferreira, S. B.; Mendonça, J. S.; Costa, M.; Rebello, F. R.; Quim. Nova 2005, 28, 77

57. Siddiqui, I. R.; Singh, P. K.; Singh, J.; J. Agric. Food Chem. 2003, 51, 7062

58. Ding, J.; Das, K.; Moereels, H.; Koymans, L.; Andries, K.; Janssen, P. A. J.; Hughes, S. H.; Arnold, E.; Nat. Struct. Mol. Biol. 1995, 2, 407.

59. Vigorita, M. G.; Ottanà, R.; Monforte, F.; Maccari, R.; Monforte, M. T.; Trovato, A.; Taviano, M. F.; Miceli, N.; De Luca, G.; Alcaro, S.; Ortuso, F.; Bioorg. Med. Chem. 2003,11, 999.

60. Goel, B.; Ram, T.; Tyagi, R.; Bansal, E.; Kumar, A.; Mukherjee, D.; Sinha, J. N.; Eur.J. Med. Chem. 1999, 34, 265.

61. Walsh, D. A.; Uwaydah, I. M.; U.S. pat. 5,061,720, 1991.

62. Allen, S.; Newhouse, B.; Anderson, A. S.; Fauber, B.; Allen, A.; Chantry, D.; Eberhardt, C.; Oding, J.; Burgess, L. E.; Bioorg. Med. Chem. Lett. $\mathbf{2 0 0 4}, 14,1619$.

63. Hrib, N. J.; Jurcak, J. G.; Eur. pat. appl. 0316723 A1, 1989; Hrib, N. J.; Jurcak, J. G.; U.S. pat. 4,933,453, 1990; Hrib, Nicholas J.; U.S. Patent 5,034,392, 1991; Hrib, N. J.; Strupczewski, J. T.; Jurcak, J. G.; Bordeau, K.; U.S. Patent 5,041,445, 1991

64. Enomoto, M.; Kojima, A.; Komuro, Y.; Morooka, S.; Aono, S.; Sanemitsu, A.; Mitzutani, M.; Tanabe, Y.; U.S. pat. 4,992,455, 1991 